\title{
Sequential activation of premotor, primary somatosensory and primary motor areas in humans during cued finger movements
}

Hai Sun ${ }^{\mathrm{a}, \mathrm{b}}$, Timothy M Blakelyc ${ }^{\mathrm{c}}$, Felix Darvas ${ }^{\mathrm{b}, \mathrm{c}}$, Jeremiah D. Wander ${ }^{\mathrm{c}}$, Lise A. Johnson ${ }^{\mathrm{b}, \mathrm{d}}$, David K. Su ${ }^{\mathrm{b}}$, Kai J. Miller ${ }^{\mathrm{e}}$, Eberhard E. Fetz ${ }^{\mathrm{d}, \mathrm{ef}}$, Jeffery G. Ojemann ${ }^{\mathrm{b}, \mathrm{c}, \mathrm{g}, \mathrm{g}}$

${ }^{a}$ Department of Neurological Surgery, Oregon Health \& Science University, Portland, OR, USA

${ }^{b}$ Department of Neurological Surgery, University of Washington, Seattle, WA, USA

${ }^{c}$ Department of Bioengineering, University of Washington, Seattle, WA, USA

${ }^{\mathrm{d}}$ The Center for Sensorimotor Neural Engineering, Seattle, WA, USA

${ }^{\mathrm{e}}$ Neurobiology and Behavior Degree Program, University of Washington, Seattle, WA, USA

${ }^{\mathrm{f}}$ Department of Physiology and Biophysics, University of Washington, Seattle, WA, USA

${ }^{\text {g}}$ Seattle Children's Hospital, Seattle, WA, USA

Corresponding Author:

Hai Sun, MD, PhD

Department of Neurological Surgery

Oregon Health \& Science University

Center for Health \& Healing, CH8N

3303 SW Bond Ave.

Portland, OR 97239, USA

Tel.: +1-503 494-4314

Fax: +1-503-346-6810

E-mail: hai.sun@bnaneuro.net 


\begin{abstract}
Objective: Human voluntary movements are a final product of complex interactions between multiple sensory, cognitive and motor areas of central nervous system. The objective was to investigate temporal sequence of activation of premotor (PM), primary motor (M1) and somatosensory (S1) areas during cued finger movements.
\end{abstract}

Methods: Electrocorticography (ECoG) was used to measure activation timing in human PM, S1, and M1 neurons in preparation for finger movements in 5 subjects with subdural grids for seizure localization. Cortical activation was determined by the onset of high gamma (HG) oscillation $(70-150 \mathrm{~Hz})$. The three cortical regions were mapped anatomically using a common brain atlas and confirmed independently with direct electrical cortical stimulation, somatosensory evoke potentials and detection of HG response to tactile stimulation. Subjects were given visual cues to flex each finger or pinch the thumb and index finger. Movements were captured with a dataglove and time-locked with ECoG. A windowed covariance metric was used to identify the rising slope of HG power between two electrodes and compute time lag. Statistical constraints were applied to the time estimates to combat the noise. Rank sum testing was used to verify the sequential activation of cortical regions across 5 subjects.

Results: In all 5 subjects, HG activation in PM preceded S1 by an average of $53 \pm 13 \mathrm{~ms}(P=0.03)$, PM preceded M1 by $180 \pm 40 \mathrm{~ms}(P=0.001)$ and S1activation preceded M1 by $136 \pm 40 \mathrm{~ms}(P=0.04)$. Conclusions: Sequential HG activation of PM, S1 and M1 regions in preparation for movements is reported. Activity in S1prior to any overt body movements supports the notion that these neurons may encode sensory information in anticipation of movements, i.e., an efference copy (EC). Our analysis suggests that $\mathrm{S} 1$ modulation likely originates from PM.

Significance: First electrophysiological evidence of efference copy EC in humans.

Keywords: Motor system, efference copy, electrocorticography, forward model, somatosensory integration, sensory feedback, and brain computer interface. 


\section{Introduction}

Humans have remarkable ability to perform complex voluntary movements. These movements are results of complex interactions between different cortical areas including the primary motor cortex (M1), the supplementary motor area (SMA), the premotor cortex (PM), and the primary somatosensory cortex (S1) (Day et al., 1989, Ghez et al., 1997, Kutas and Donchin, 1974, Riehle and Requin, 1993, Rosenbaum, 1980, Wise, 1985 )(Rao et al., 1993)(Christensen et al., 2007). Attempts have been made to capture the timing of activation of these areas in order to elucidate their roles in motor generation and control. For example, single neuron recordings in nonhuman primates showed the pathway by which visually guided reach movements are generated begins in the extrastriate visual cortex (Felleman and Van Essen, 1991) and passes through the parietal reach region (PRR) and area 5 to the dorsal premotor cortex (PMd) and then to the primary motor cortex (Johnson et al., 1991)(Wise et al., 1997). In rhesus macaques, Soto and Fetz reported that cells in primary somatosensory cortex fire prior to active movements. This phenomenon demonstrated that $\mathrm{S} 1$ is an integral part of movement generation (Soso and Fetz, 1980). Also working with nonhuman primates, Nelson recorded changes in discharge rates in S1 that occurred early before movement onset; and hypothesized that S1 is modulated by other cortical region(s) before any movements take place (Nelson, 1987). In these animal studies it was demonstrated that $\mathrm{S} 1$ neurons were activated prior to the actual movements, which supported a concept important for somatosensory integration during voluntary movements known as the efference copy (EC).

Simply stated, an EC is an internal prediction of the sensory consequence of a volitional movement. The EC is thought to serve two important functions. Firstly, EC is used by the brain 
to distinguish sensory signals produced by one's own actions from those generated by external stimuli (Wolpert et al. , 1995, Helmholtz, 2005). This influences sensations experienced during active movements (Chapman et al. , 1987, Blakemore et al. , 1998). Secondly, EC assists in control of movements when peripheral sensory feedback cannot reach the central nervous system fast enough (Miall et al. , 1996). EC is an essential component of an internal forward model of motor planning and control, which has gained prominence due to emergent evidence from psychophysical and electrophysiological studies (von Holst, 1954, Jordan et al. , 1992, Miall et al. , 1996). Electrophysiological evidence is however, confined to nonhuman primate models. Studies using single neuron recordings in these models have shown a change in firing rates of cells in S1 cortex prior to active movements (Soso et al. , 1980, Nelson, 1987), and the timing of this change is consistent with encoding of EC signals. A pathway from the superior colliculus to the frontal eye field via the thalamus has also been identified for conveying EC signals related to eye movements in monkeys (Sommer et al. , 2002a). Evidence of EC has also been reported in a study of vestibular nuclei neurons encoding head angular velocity during passive and active head turns in Rhesus monkeys (Roy et al. , 2001). Another phenomenon related to EC is the suppression of auditory response in self-generated sounds (Ford et al. , 2001, Poulet et al. , 2002).

Electrophysiological evidence of EC in humans is currently lacking. The timing of neural activation in different cortical regions relative to movement has been studied in humans using different imaging modalities. Using near-infrared spectroscopy (NIRS) signals and movementrelated cortical potentials (MRCP), Sato et al., showed in humans that MRCP started approximately $1.5 \mathrm{~s}$ before electromyogram (EMG) change of finger movement. The NIRS data showed bilateral prefrontal cortex activations at approximately $0.5 \mathrm{~s}$ before EMG onset and 
bilateral dorsal premotor cortex activations at approximately $0.6 \mathrm{~s}$ before EMG onset. The hand area of the sensorimotor cortex was activated left-dominantly, peaking at approximately $3.7 \mathrm{~s}$ after EMG onset (Sato et al. , 2012). Using magnetoencephalography (MEG) in humans, Nishitani and Hari reported, during imitation, left inferior frontal cortex led M1 in activation by 0.05 to $0.25 \mathrm{~s}$ (Nishitani et al. , 2000, 2002). These modalities lacked the temporal resolution and sptial coverage to accurately study the interactions of different cortical regions.

Here, we used electrocorticography (ECoG) to record the timing of neuronal activation in premotor (PM), primary motor (M1) and S1 neural populations relative to cued finger movements in humans. An implanted subdural ECoG grid covers different cortical regions and is capable of detecting changes in activity produced by neuronal ensembles focal enough to distinguish somatotopic areas (Miller et al. , 2007, Kubánek et al. , 2009). Changes in high gamma $(\mathrm{HG})$ power $(>70 \mathrm{~Hz})$ in ECoG have been shown to correlate with local cortical activity (Brindley et al., 1972, Crone et al. , 1998, Yoshor et al. , 2007, Ray et al., 2008, Mesgarani et al. , 2012). These broadband changes capture functionally specific cortical activity with a temporal resolution of milliseconds (Pei et al., 2011, Swann et al. , 2013).

\section{Materials and Methods}

\subsection{Subjects and Recordings}

This study was a retrospective analysis of previously recorded ECoG data. Consecutive subjects were recruited at Harborview Medical Center and Seattle Children's Hospital between December 2010 and December 2012. Subjects gave written informed consent according to a protocol approved by the institutional review boards at both institutions. Five ( 1 female and 4 
male) subjects were included in this study. The inclusion criteria included: 1) subjects had electrode coverage of at least two of the three cortical areas of interest, i.e., PM, M1, and S1, based on cortical mapping, and 2) subjects responded to the visual cue in a timely fashion, i.e., performing finger movements during the action phase of the task and waiting for the next cue during the resting phase. One subject was excluded for inability to respond timely to the movement cue. All subjects had ECoG grids placed for extended clinical monitoring and localization of seizure foci, in the course of the treatment of medically-refractory epilepsy. All electrode grids were constructed of $2.4 \mathrm{~mm}$ diameter exposed platinum-iridium pads embedded in silastic (AdTech, Racine, WI.) In 4 subjects (subjects 1-3 and 5), the electrode spacing was 10 $\mathrm{mm}$ while the electrode spacing the grid used in subject 4 was $7 \mathrm{~mm}$. Electrodes were arranged as $8 \times 8$ or $4 x 8$ array. ECoG signals were obtained by splicing the clinical system's recording sampled at either at $1.2 \mathrm{kHz}$ or $2.4 \mathrm{kHz}$ using either SynAmps2 (Neuroscan, El Paso, TX, USA) or g.USBamp (Guger Tec, Graz, Austria).

\subsection{Motor Tasks}

Subjects perform a screening motor task, which involves gross motor movements of either their tongue or their hand contralateral to the side of the electrode placement for $3 \mathrm{~s}$, followed by a similar period of rest. This process was repeated between 10 and 30 times for each motion. Based on the appearance of movement-induced HG activities among electrodes and their relationship with gyral anatomy, we determined the electrode coverage of cortical regions. Only subjects with electrode coverage of at least two of the three cortical regions were asked to perform the cued finger movement task. 
In the cued finger task, subjects were shown a silhouette of a hand on a computer screen at the start of each trial (Fig. 1). The finger silhouette instructed the subject to flex the same finger that was highlighted with a unique color or make a pinching motion when both thumb and index fingers were highlighted. After $2 \mathrm{~s}$ of repetitive motion, the screen became blank signaling a rest period of $1 \mathrm{~s}$. This was repeated between 10 to 30 times for each finger movement type. The trial types were alternated randomly so that each trial remained novel to the subject. A 22-degree-offreedom dataglove (CyberGlove Systems, San Jose, CA) was used to capture finger movements sampled at $1.2 \mathrm{kHz}$. Neural signals and dataglove samples were collected and time-locked by the BCI2000 recording suite (Schalk et al., 2004).

\subsection{Three independent methods of cortical mapping}

Three independent methods for cortical mapping were utilized. In method 1, preoperative MR images were co-registered with postoperative CT scans using the statistical parametric Mapping software package (Penny, 2006). The cortical surface was first segmented from preoperative MR images using the FREESURFER environment (Dale et al. , 1999). The electrode positions were calculated by segmenting them from a high-resolution postoperative CT scan registered with the preoperative MR imaging (Hermes et al. , 2010). Each individual MR volume was registered to the Talairach brain atlas to allow an accurate localization of the electrode position (Talairach et al. , 1988). The electrodes projected to the precentral gyrus of the common atlas are M1 electrodes (Brodmann area 4), whereas the electrodes projected to the postcentral gyrus are S1 electrodes (Broadmann areas 1-3). Electrodes located anteriorly to the M1 electrodes are PM electrodes. 
In method 2, the accuracy of somatosensory mapping based on the common brain atlas, was verified by demonstrating that ECoG signals recorded by the electrodes in this region responded to sensory stimulation of the contralateral hand with increased HG power. In this experiment, the subject's hand contralateral to the grid implant site was repeatedly touched with an object. A signed squared cross-correlation value was calculated by comparing the distribution of HG power during hand stimulation with the distribution during rest (Miller et al. , 2007); in subjects 1 to 4 .

In method 3, attempts to record somatosensory evoked potentials (SSEPs) at the relevant electrodes and performed electrical cortical stimulation (ECS) at these electrodes was undertaken. SSEPs map the sensorimotor cortex by eliciting intrinsic cortical physiological responses to peripheral nerve stimulation at a rate of $5.1 \mathrm{~Hz}$, with a pulse width of $200 \mathrm{~ms}$ and a current of $6 \mathrm{~mA}$, most often of the median or posterior tibial nerves. The SSEP N20 waveform is recorded over the contralateral somatosensory cortex, whereas a P20 waveform with the opposite polarity is recorded over the primary motor cortex (Legatt et al., 2000, Ikeda et al. , 2008). Referential recordings from adjacent electrodes over the pre- and postcentral gyri produce a "phase reversal" between the N20 and the P20, marking the site of the central sulcus, which is directly underneath or a few millimeters anterior to the area of highest amplitude. Electrode cortical stimulation was performed in awake subjects at the bedside by using an Ojemann stimulator (Integra, Plainsboro, NJ) to elicit movements by delivering up to $12 \mathrm{~mA}$ of current to pairs of implanted electrodes, and patients were monitored for movement or reproducible sensations of the hand, face, or leg.

\subsection{Power spectral analysis}


Offline data processing was performed with custom MATLAB software (Natick, MA). All ECoG data were re-referenced with respect to the common average. To minimize the trial-bytrial variability in task performance, the continuous recordings were segmented into epochs centered on movement onset, as measured by the dataglove. Time-frequency dynamic spectra were made using both a wavelet and the Hilbert transform approach. The wavelet approach used a Morlet wavelet (Goupillaud et al. , 1984) to convolve with the voltage time-series to generate a time-frequency estimate for every frequency bin between 1 to $200 \mathrm{~Hz}$. This approach allows for investigation of changes in the power spectrum density across all frequencies. For every electrode, we calculated statistically corrected activation weights ( $\mathrm{z}$ scores) by comparing the power density during the activity phases with resting phases (Miller et al., 2007).

Since an increase in $\mathrm{HG}$ power in ECoG has been shown to correlate with local cortical activity (Brindley et al. , 1972, Crone et al. , 1998), we employed the Hilbert transform approach to construct a time course of the HG power for all electrodes. The Hilbert transform of a voltage time-series produces a complex signal. The HG power time series can be computed by taking the norm squared of the Hilbert transform. Similar to wavelet approach, a signed squared crosscorrelation value was calculated by comparing the distribution of $\mathrm{HG}$ power during movement with the distribution during rest(Miller et al. , 2007). The statistical significance associated with the HG power distribution was estimated using a balanced, unpaired $t$ test. This allows selection of electrodes by applying a threshold. This step helped focus attention on the areas with HG activations.

\subsection{A short-time windowed covariance (STWC) metric}


With the HG power time-series, the time differences of neuronal activation between two electrodes could be estimated. This was achieved by capturing the rising phases of HG power of both electrodes and computing the time lag between these two events. A STWC metric was employed for this task (Blakely et al. , 2014). To reduce computational cost, the HG power time series was down sampled by a factor of 10 . To avoid aliasing, the time series were filtered with a low pass filter with a cutoff frequency of $5 \mathrm{~Hz}$ before down sampling. Each down-sampled signal was then normalized before the computation of the STWC metric (van Albada et al. , 2007). A covariance calculation is performed with a small sample window of the source electrode $x$ centered on sample $t$ with multiple windows of a destination electrode $y$ :

$$
Q(x, y, t, \tau, \delta)=\sum_{i=t-\frac{\tau}{2}}^{t+\frac{\tau}{2}} \frac{\left(x_{i}-\bar{x}\right)\left(y_{\delta+i}-\overline{y_{\delta}}\right)}{\tau+1}, t \in[1, n] \text { and } \delta \in[-\Delta, \Delta] \text {, where } \tau \text { is the window }
$$

size, $n$ is the number of ECoG samples in a trial and $\delta$ is the number of samples by which the window on $y$ is shifted (a negative value means the window on $y$ is shifted ahead of window on $x$ in time, whereas a positive value indicates a delay and $\Delta$ is the maximum of $\delta$ ). For a pair of ECoG electrodes and a fixed window size $\tau, Q$, the covariance, becomes a function of $t$ and $\delta$. For all subjects, we selected the number of samples in a half second to be the fixed window size $\tau$ and $\Delta$. The selected windows in channel $y$ are the same length of the channel $x$ 's window but shifted from $-\Delta$ to $\Delta$, giving $(2 \times \Delta+1)$ covariance measures for each sample point $t$. The covariance values were then averaged over trials. For each paired of electrodes, the STWC metric generates a two dimensional map, $Q_{0}(t, \delta)$, where $t \in[1, n]$ and $\delta \in[-\Delta, \Delta]$.

\subsection{Statistical significance of each value in $Q_{0}$}


Statistical significance of each value of $Q_{0}$ was tested by applying a bootstrap method using surrogate signals generated with phase randomization (Nur et al. , 2001). For two ECOG signals from a pair of electrodes, phase randomization was performed 500 times for each signal generating 500 pairs of surrogate signals. Applying the STWC metric to each pair of surrogate signals led to a new two dimensional map $Q_{i}$, where, $i \in[1,500]$. By performing the phase randomization, we destroyed any temporal relationships existing between two ECoG frequency distributions and hence established a NULL hypothesis that there is no temporal relation between the two HG power time series. The statistical significance of each covariance value in $Q_{0}$ can be estimated by comparing it with the 500 trials in $Q_{i}$. Multiple testing when computing $P$ value for each covariance value was also adjusted (Nichols, 2012). A covariance value with an associated $P$ value $<0.05$ was deemed significant.

\subsection{The time lag of $H G$ activation between two electrodes}

The task of estimating the time lag between the HG onsets in two electrodes involves finding statistically significant $Q$ values and their corresponding $t$ 's and $\delta$ 's. Having identified all statistically significant $Q$ values $(P<0.05)$ and their corresponding $t$ 's and $\delta$ 's, $Q$-value weighted means of both $t$ and $\delta, \bar{t}$ and $\bar{\delta}$, respectively were computed. $\bar{t}$ is now the time point where electrode $x$ is activated in response to motor command and $\bar{\delta}$ is the lag by which electrode $y$ becomes activated relative to electrode $x$ (a negative $\bar{\delta}$ indicates electrode $y$ activates before electrode $x$ whereas a positive $\bar{\delta}$ indicates the opposite). Both values can then be converted to time measurements by multiplying the sampling frequency and with the expressed relative to the onset of finger movements. 


\subsection{Statistical significance of the STWC-derived time lag of HG activations}

To further limit the influence of noise, the statistical significance of a time lag of HG activation of two electrodes was estimated. The sequence of $\mathrm{HG}$ activation for any electrode pair can be estimated from the STWC metric or from the timing of activation of each electrode in the pair relative to a third electrode. These multiple estimates of the activation sequence between two electrodes were then subjected to binomial testing. The final activation time of an electrode is the mean of all statistically significant estimates $(P<0.05)$ for this electrode.

\subsection{The timing of $H G$ activation of each electrode}

The estimation of activation time for an electrode site was therefore based on the statistically selected STWC results. Each STWC metric yielded one estimate of activation time for two electrode sites, $x$ and $y$, involved in computation. The HG activation time of site $x$ is $\bar{t}$ whereas the activation time of site $y$ is $\bar{t}+\bar{\delta}$. The final activation time of an electrode site was then calculated by finding the mean of all statistically significant estimates for this site. Of the four subjects with reliable dataglove recordings, this time of activation was calculated relative to the onset of movements. In one subject for whom the dataglove recording was suboptimal, the time of activation was calculated relative to the onset of cues.

\section{Results}

Five subjects (aged 18-54 years; 1 female) with sensorimotor ECoG grid coverage met the study inclusion criteria. Demographics of these subjects are listed in Table 1. The composite grid coverage for all 5 subjects is shown in Fig. 2. 


\subsection{Changes in power spectral density}

The primary study goal was to quantitatively estimate the relative timing of cortical activations in PM, S1, and M1 neurons, as measured by HG activity. Subjects were visually cued to flex particular digits or perform a pinch between thumb and forefinger. Signal dynamics were documented by the temporal and spatial characteristics of power spectral density (PSD) changes of ECoG signals, (Fig. 3). In cued finger movements, these averaged PSDs showed an increase in $\mathrm{HG}$ power associated with movement onset and a decrease in $\beta$ power $(13-30 \mathrm{~Hz})($ Crone et al. , 1998, Miller et al. , 2007). Notably, spectral changes began before finger movements in all three regions. Moreover, these changes occurred sequentially in different cortical areas, beginning with PM, followed by S1, and ending with M1 sites. Finger movements were recorded with a dataglove for 4 of the 5 subjects. Sequential changes in PSD among electrodes from three brain regions were observed in other somatotopically related electrodes across other individuals.

\subsection{High gamma activation times estimated with a covariance metric}

First the electrodes that had HG broadband changes associated with the finger movements were identified. For each electrode, the signed squared cross-correlation value of $\mathrm{HG}$ change associated with movements by comparing the distribution of $\mathrm{HG}$ power during movement with the same distribution during rest (Miller et al. , 2007) was estimated. Only electrodes with statistically significant increase in $\mathrm{HG}$ power were included in the analysis. A short time windowed covariance (STWC) metric was used to identify the statistically significant STWC values $(P<0.05)$ yield the time lag of HG activation between a pair of sites and a specific timeframe when the correlation occurs. Combining these two values leads to two HG activation times, one for each electrode of the pair. An example of estimating HG activation times of two 
electrode sites using STWC is depicted in Fig. 4. Note the PSD changes of these two electrodes related to finger movements shown in Fig. 3.

\subsection{Cortical Mapping}

This step identified electrodes in three cortical regions, PM, S1, and M1. Three independent cortical mapping methods yielded congruent results. Results of brain mapping from all 5 subjects are shown in Fig. 5. HG activation times for all electrodes with sufficient HG response to the finger movements using STWC were computed. The estimated HG activation times of each selected electrodes from all subjects are shown in Fig. 6. Note in all subjects, the HG activation began with PM, followed by S1, and lastly by M1 sites after the electrodes have been assigned to one of the sites based on cortical mapping.

\subsection{The activation time of each cortical region}

The activation time of each region was computed for each subject by determining the mean HG activation times among the sites localized to each region, Fig. 7 and Table 2. Among the 4 subjects (subjects 1,2,4, and 5) who had electrode coverage of all three cortical regions, the HG activation began in PM, was followed by S1 and lastly by M1 sites. In subject 3, the HG activation in PM led that in M1 neurons.

To demonstrate that finger movement type has little influence on the results, subject 5 was asked to perform the same motor task with each finger movement repeated 30 times in random order. The activation time of each of the three cortical regions was then computed using the same method outline above but with only signals from the same finger movement included in the 
analysis. As shown in Fig. 8, the activation of $\mathrm{S} 1$ consistently preceded that of $\mathrm{M} 1$ in all six types of finger movements.

\subsection{Time lags of the cortical activation among three regions}

The time lag of HG activation between two cortical regions was calculated by finding the difference of the mean of $\mathrm{HG}$ activation time of electrodes in each region. The time lag of cortical HG activation was calculated for each subject and then averaged across subjects. The HG activation in the $\mathrm{PM}$ region leads that in the $\mathrm{S} 1$ region by an average of $53 \pm 13 \mathrm{~ms}(P=$ 0.03, paired-sample t-test), PM leads M1 by $180 \pm 40 \mathrm{~ms}(P=0.001)$ and activation in S1 leads M1 by $136 \pm 40 \mathrm{~ms}(P=0.04$; Fig. 9$)$.

\section{Discussion}

The purpose of this study was to characterize the relative activation times of three cortical regions: PM, S1, and M1, in preparation of cued finger movements in human subjects. Increase in $\mathrm{HG}$ activities was used as a measure of the cortical activation and estimated the activation time of these three regions in relation to each other and relative to the movements. Using a datadriven approach with a novel covariance metric and a series of statistical constraints, a significant temporal relationships of changes in $\mathrm{HG}$ power demonstrated the sequential $\mathrm{HG}$ activation of PM, S1 and M1 regions in preparation for movements.

\subsection{Electrophysiological evidence of efference copy in humans}

A significant study result was the identification of early involvement of sensory cortex in motor planning. S1 cortex was activated not only before peripheral muscles and movements, but 
also before the activation of M1 cortex. This activation of S1 prior to motor efference, and well in advance of any sensory input, is consistent with models of movement that require prediction of sensory changes prior to a planned (finger) movement, i.e. EC. In humans, psychophysical studies have demonstrated that sensory predictions precede motor acts (Johansson et al. , 1992, Flanagan et al. , 2003, Gritsenko et al. , 2007) and lesion studies have demonstrated the importance of EC in movement control (Duhamel et al. , 1992, Gaymard et al. , 1994, Leiguarda et al. , 2000). A study conducted with functional magnetic resonance imaging (fMRI) in humans has shown that $\mathrm{S} 1$ neurons are active in voluntary movements in the absence of peripheral sensory inputs (Christensen et al. , 2007). In the same study, Christensen et al., hypothesized that $\mathrm{S} 1$ is likely modulated by the premotor area based on the correlation of blood oxygen level dependent (BOLD) signals in these two regions (Christensen et al. , 2007). Here, we use human electrophysiological evidence of the temporal characteristic of S1 activation to support the notion of both EC and the internal forward model.

Electrophysiological evidence of EC is not only important for understanding human motor physiology, EC is also implicated in human cognitive and behavioral functions. The existence of EC implies that the sensory system is not only influenced by environment but is also modulated by the brain. It is through EC one can distinguish the owner of a motor action, i.e., the sense of agency (Farrer et al. , 2003, Tsakiris et al. , 2006). EC explains why we cannot tickle ourselves (Blakemore et al. , 2000).

While we believe that we have captured neuronal activities related to EC, it is possible that in the experiments described only one particular type of EC at a specific time point, i.e., the moment immediately before the movement onset is captured. As shown in previous studies, 
neuronal firing can either increase or decrease prior to the movement depending on the regions in S1 (Nelson, 1987, Chapman, 1994). The interplay between the EC and peripheral sensory afferent is also complex, which can lead to different sensory perceptions by the subject. Comparing with externally generated stimuli, some studies reported reduction (i.e. gating) (Blakemore et al. , 1998, Baess et al. , 2009) of neuronal activities in S1 following self-initiated motor acts, while others suggest enhancement of these activities (Hughes et al. , 2011).

\subsection{PM, rather than M1, modulates S1 before movements}

This study demonstrates the existence of EC-related S1 activation and also shows that this activation precedes the activation of M1 and is preceded by the activation of PM. The timing of these events suggest that, immediately prior to movements, $\mathrm{S} 1$ neurons are likely modulated by PM rather than M1 neurons. Christinsen et al., have proposed this relationship based on the correlation of BOLD signals in fMRIs between these two regions during human voluntary movements (Christensen et al. , 2007). The results obtain in nonhuman primates are different. In two separate studies conducted in awake macaques, the timing of neuronal activation in precentral (Fetz et al. , 1980) and postcentral (Soso et al. , 1980) gyri was documented during active and passive elbow movements. Similar to our observations, they showed that the neurons were activated in both precentral and postcentral gyri before EMG activities. In these experiments, the average onset of neurons in precentral gyri preceded average activation of postcentral neurons. Future experiments that utilize the same recording modality and similar motor tasks need to be conducted both in humans and primate models to elucidate the cause for this discrepancy. A study of the modulation of cortical potentials evoked by electrical stimulation of a cutaneous nerve in an instructed delay task showed early modulation in PM and 
M1 prior to the active movement, consistent with the predictions of an EC signal (Seki et al. , 2012).

It is important to emphasize that the phenomenon reported here is limited to the transient period immediately prior to finger movements in these particular motor tasks. S1 neurons can be modulated by M1 neurons in other settings. For example, a study has shown that electrical stimulation of M1 neurons changes the firing patterns of S1 neurons and hence the sensory perception (Tsubokawa et al. , 1991). In addition, the motor task we used is based on visual cues. For future studies it will be important to investigate timing of cortical activations during spontaneous and reactive (cued) movements. Type of cues may also influence pattern of cortical activation. Similar studies can be conducted with auditory cues.

The sequential activation of PM, S1 and M1 neurons documented here suggests a possible pathway by which the EC is transmitted from PM to S1 rather than from M1 to S1. The ECrelated activation is not limited to these two regions. A fMRI study showed that the forward model of motor control involved the cerebellum (Kawato et al. , 2003). One well-studied pathway for visually guided reach movements begins in the extrastriate visual cortex (Felleman et al. , 1991) and passes through the parietal reach region (PRR) and area 5 to the dorsal premotor cortex and then to the primary motor cortex (Johnson et al., 1991, Wise et al. , 1997). Other structures possibly involved include cortical regions such as supplementary motor area (SMA) (Fried et al. , 2011) and subcortical structures such as basal ganglia and thalamus. Our methodology, when combined with depth electrodes, can be applied to documenting interactions of these structures to further elucidate the pathway for EC.

\subsection{Implications for neuroprothesis design}


Better understanding of how sensory information is integrated in initiation and control of movements is important for developing robust neuroprostheses. Historically, brain computer interface (BCI) device development has focused on using brain activities in the conventional motor cortices, although volitional control of postcentral cells has also been demonstrated (Moritz et al. , 2011). Evidence has emerged that both pre and post-central gyri continue to be activated in individuals with chronic spinal cord injury during attempted movements (Shoham et al. , 2001, Cramer et al. , 2005, Hotz-Boendermaker et al. , 2008). The study confirms that sensory cortex activation is an integral part of motor planning that is independent of feedback from peripheral afferents. These signals may still be present in individuals with disease processes that selectively affect M1 such as focal ischemic stroke or motor neuron diseases. A natural extension of this study is to investigate interactions of these cortical regions during performance of imagined movements.

The presence of EC-related S1 activation provides a potential means for the implementation of somatosensory feedback in BCI applications. Currently application of BCI mostly relies on visual feedback information to users. There is, however, an increasing need to include other forms of sensory feedback to BCI devices (Schwartz et al. , 2006, Nicolelis et al. , 2009, Johnson et al. , 2013). Some groups have proposed adding sensory modalities such as vibrotactile (Chatterjee et al. , 2007), proprioceptive (Ramos-Murguialday et al. , 2012), and auditory (Nijboer et al. , 2008) feedbacks to BCI devices to increase their efficacy and reduce user training time. By identifying the electrophysiological correlate of the EC using ECoG, we have separated anticipatory sensory signals produced by $\mathrm{BCI}$ users from those generated by external stimuli as potential inputs for BCI devices. Comparison of these two signals in real time may lead a sensory feedback paradigm. 


\subsection{Study limitations}

It is important to consider the limitations associated with human ECoG studies. All subjects included here were undergoing surgical treatment of medically intractable epilepsy. The intracerebral pathology involved with this subject population should be kept in mind when making extensive generalizations of the study results.

Study analysis revealed that the sequence of activation from PM, then S1 and finally M1 is consistently preserved across subjects. Consistency in results was achieved when human physiological noise data including ECoG and dataglove recordings were averaged over a large number of repeated finger movements (> 90 trials). For example, despite a high sampling rate $(1.2 \mathrm{kHz})$, it is not possible to accurately define finger position from dataglove recording during one movement trial. When these recordings were averaged over a large number trials, a definite pattern of finger movement emerges, Fig. 3.

In subject 5, our results showed that PM lagged behind S1 in activation when only signals from the middle finger movements (30 trials) were included in the analysis, Fig. 8. We believe that this aberrant result likely reflects the impact of signal noise in analysis rather than true timing of activation among these cortical regions.

We defined PM electrodes as those anterior to M1. PM subdivisions have been well studied in nonhuman primates and include areas such as PM dorsal rostral; PMDr (F7), PM dorsal caudal; PMDc (F2), PM ventral, dorsal; PMVr (F5), PM ventral, caudal; PMVc (F4) and frontal eye field (FEF) (Weinrich et al. , 1984). The function of each region is complex and has undergone intense investigation. Sommer and Wurtz demonstrated that FEF is involved in EC 
during eye movements (Sommer et al. , 2002b). Depending on the grid coverage in each patient, the number and the actual location of a PM electrode vary among these PM subdivisions. Additionally, the intracerebral pathology involved with this subject population makes it unreliable to infer the function of a small cortical region based on anatomy alone.

Our analysis revealed that the actual timing of cortical activations varied considerably among the 5 subjects, Table 2. The most essential study finding is the relative temporal relationship of $\mathrm{HG}$ onsets among the three cortical regions rather than the absolute timing of each event. A factor that may contribute to the variability in estimates of actual onset of HG power may be the subject's ability to react to the visual cue. This ability is affected by but not limited to factors such as patient age, medical history, underlying brain pathology, medications, time of the day, and hospital environments. Together these variables likely account for some of the variability among subjects. A second factor is our method of using the STWC metric for the estimation of timing of HG onset contributes to the variability of actual activation times. The STWC metric was designed and implemented and associated with statistical constraint to minimize the impact of noise on electrophysiological signal results. By design, this metric is more robust in estimating the time lag between changes in HG power in two cortical regions(Blakely et al. , 2014) but is less accurate in estimating the time lag between the change in HG power and finger movements, which leads to more variability in the results. The inclusion of additional study subjects could certainly address these study limitations, although our conclusions would likely remain the same.

\section{Conclusion}


We have used ECoG to measure the time of activation in human PM, M1 and S1 neurons in preparation for finger movements. These measurements have revealed that the onset of finger movements is preceded by the increase in $\mathrm{HG}$ power in all three cortical regions. Furthermore, the increase of HG power begins in PM and is followed by S1 and then at last by M1. The timing of these events suggests that the anticipatory sensory activation of S1 is likely related to the EC and this activation of $\mathrm{S} 1$ is a result of modulation by PM rather than by M1. These findings provide the first electrophysiological evidence of EC in humans and strengthen the argument for an internal forward model for the control of human voluntary movements. Collectively, the results further better understanding of human motor and cognitive function and can help unlock the answers to disorders such as motor apraxia and mental illness such as schizophrenia. Additionally, a better understanding of how sensory information is integrated in initiation and control of movements is a key component in the development of robust neuroprosthesis for stroke or spinal cord injury patients.

\section{Conflict of Interest}

The authors have no conflicts to report.

\section{Acknowledgements}

This project was supported by the Christopher Getch Fellowship awarded by the Congress of Neurological Surgeons (HS) and the National Institute of Health (NIH) R01 grant NS065186-01 (JGO). Additional support from NIH T90 DA023436-02 (JDW), NIH-National Institute of Neurological Disorders and Stroke R25 grant NS079200 (DS), the National Science Foundation grant EE-1028725 (LAJ), and the Keck Foundation (EEF and JGO). The authors thank Shirley McCartney, Ph.D., for editorial assistance and Andy Rekito, M.S. for assistance with the figures. 


\section{Figure Legends}

Fig. 1. Cued finger movement task. Subjects were shown a silhouette of a hand on a computer screen. At the start of each trial, subjects were instructed to flex the finger that was highlighted with a unique color on the hand silhouette or to make a pinching motion when both thumb and index fingers were highlighted. After $2 \mathrm{~s}$ of repetitive motion the screen became blank, signaling a rest period of $1 \mathrm{~s}$. This was repeated between 10 to 30 times for each finger and same number of trials for the pinch. The trial types were alternated randomly so that each trial remained novel to the subject. Finger movements were recorded with a dataglove and time-locked with ECoG signals.

Fig. 2. The electrocorticography coverage of five subjects. All subjects had the 64-channel subdural electrode coverage of the sensorimotor area. This was achieved with two $4 \times 8$ electrode grids (yellow) in subject 2 and with one $8 \times 8$ electrode grid in the others. All electrode locations were registered, projected and plotted on the surface of right hemisphere of a common brain atlas. Only electrodes located at the corner of a grid are displayed here. Subjects 2 and 3 had left-sided coverage. All electrode grids were constructed of $3 \mathrm{~mm}$ diameter platinum pads embedded in silastic. In 4 subjects (subjects 1-3 and 5), the electrode spacing was $10 \mathrm{~mm}$ while the electrode spacing the grid used in subject 4 (green) was $7 \mathrm{~mm}$. sub, subject.

Fig. 3. Sequential changes in power spectral density (PSD) among electrodes from three regions of the brain. The results from all 5 subjects are included. The subplot from subject 2 is shown in larger plot on the first row for better illustration. In each subplot, on the left; three electrodes from three different cortical regions, PM, S1, and M1 (except for subject 3, who has no electrode coverage of S1). On the right, the averaged power spectral density map shows that all three electrode sites exhibit decreased beta rhythms and increased broadband high gamma associated with movement. These changes all began before the movement (shown by averaged dataglove tracing over trials). Furthermore the broadband 
changes in PM electrodes occurred first and were followed by similar changes in S1 then M1. CS, central sulcus, SF, sylvian fissure, IHF, interhemispheric fissure.

Fig. 4. An example of estimated $\mathrm{HG}$ activation times for a pair of electrodes using the short-time windowed covariance (STWC) metric. a. The location of the two electrodes on the brain. b. The two normalized HG power time series averaged over trials. c. The 2-D map $Q(t, \delta)$ after statistically insignificant covariance values has been set to zero. D. Averaged dataglove tracing over trials. Computing a weighted centroid of all statistically significant covariance values leads to two time estimates $\bar{\delta}$ and $\bar{t}$. $\bar{\delta}$ is the estimated time lag of the HG activation between these two electrodes. Here the $\bar{\delta}$ has a positive number, which indicates the S1 site activates earlier than the M1 site. $\bar{t}$ is the HG activation time of the $\mathrm{S} 1$ site relative to the onset of movements. The HG activation time of the M1 electrode relative to the onset of movements is then $\bar{t}+\bar{\delta}$. D. The averaged finger position recorded with dataglove.

Fig. 5. Confirmation of the cortical mapping. The results from all five subjects are shown here. The results from subject 2 are shown in the first row in bigger plot for better illustration. In this subplot, on the left, all 64 electrodes were registered, projected and plotted with a common brain atlas. Electrodes 40, 48 and 55 were localized to S1. In the middle, changes in HG power among all electrodes when subject's contralateral hand was touched with an object relative to resting baseline. Electrodes 40, 48 and 55 had highest increase of HG power associated with sensory stimulation of the contralateral hand. On the right, the location of central sulcus (CS) relative to the grid is again confirmed based on the electrical cortical stimulation (ECS, triangle), where cortical stimulation of electrodes 39, 47, 54, 53 and 61 produced motor response. In this subject, somatosensory evoked potentials (SSEPs, circle) yielded reliable mapping results. The results from the other subjects are shown in the panels below. In subject 3 , neither ECS or SSEPs identified any electrode covering hand motor or sensory area. The grey grid is therefore omitted for this subject. In subject 5, the confirmation was only conducted with ECS and 
SSEPS, the green grid is therefore omitted for this subject. Note that, these three different mapping techniques yielded congruent identification of central sulcus (CS) in all five subjects. Sub, subject.

Fig. 6. The activation times for all electrodes with $\mathrm{HG}$ response related to movement from all 5 subjects. The shape of each electrode symbol corresponds to its location relative to three cortical regions: The circular electrodes are from PM, the diamond electrodes are from S1 and the triangular electrodes are from M1. The color of electrodes indicates which subject they belong to. A are numbers of electrodes with sufficient change in $\mathrm{HG}$ power in each cortical regions among all 5 subjects. B and $\mathrm{C}$ are results from subject 1 to 4 and the activation times were computed relative to the onset of finger movement (at time zero). $\mathrm{C}$ is a magnified portion of B to better illustrate salient features. D. The results from subject 5 and activation times computed relative to onset of visual cue (at time zero).

Fig. 7. Sequential activation of PM, S1 and then M1. The activation time for each cortical region is the mean activation time of all electrodes (n) in the region. Subject 3 had no S1 coverage. Error bars are the standard error (SE) of the mean of the sample. No error bar is calculated for a sample of one. The activation times for subjects 1-4 were calculated relative to the onset of movement (negative values) whereas the activation times for subject 5 are relative to the onset of cue (positive values). sub $=$ subject.

Fig. 8. The activation time of three cortical regions computed for each finger movement type. The sequential action of PM, S1, then M1 were maintained in flexion of thumb, index, and little fingers and thumb-index pinch. With middle and ring finger flexion, the order of activation among the three regions was S1, then PM, and lastly M1.

Fig. 9. Time lags of cortical activation among the three cortical regions, PM, S1, and M1. a. The sequential activation of the three cortical regions; arrows originate in region that leads the region where the arrow points. b. Bar graph shows that, across all 5 subjects, the HG onset in PM leads that in S1 by an 
average of $53 \pm 13 \mathrm{~ms}(P=0.03$, paired-sample $t$-test $), \mathrm{PM}$ leads $\mathrm{M} 1$ by $180 \pm 40 \mathrm{~ms}(P=0.001)$ and $\mathrm{S} 1$ lead M1 by $136 \pm 40 \mathrm{~ms}(P=0.04)$. Error bars are standard errors of the means. 


\section{References}

Baess P, Widmann A, Roye A, Schroger E, Jacobsen T. Attenuated human auditory middle latency response and evoked 40-Hz response to self-initiated sounds. Eur J Neurosci. 2009;29:1514-21. Blakely T, Ojemann JG, Rao RP. Short-time windowed covariance: A metric for identifying nonstationary, event-related covariant cortical sites. J Neurosci Methods. 2014;222:24-33.

Blakemore SJ, Wolpert D, Frith C. Why can't you tickle yourself? Neuroreport. 2000;11:R11-6. Blakemore SJ, Wolpert DM, Frith CD. Central cancellation of self-produced tickle sensation. Nat Neurosci. 1998;1:635-40.

Brindley GS, Craggs MD. The electrical activity in the motor cortex that accompanies voluntary movement. J Physiol (Lond). 1972;223:28P-9P.

Chapman CE. Active versus passive touch: factors influencing the transmission of somatosensory signals to primary somatosensory cortex. Can J Physiol Pharm. 1994;72:558-70.

Chapman CE, Bushnell MC, Miron D, Duncan GH, Lund JP. Sensory perception during movement in man. Exp Brain Res. 1987;68:516-24.

Chatterjee A, Aggarwal V, Ramos A, Acharya S, Thakor NV. A brain-computer interface with vibrotactile biofeedback for haptic information. J Neuroeng Rehabil. 2007;4:40.

Christensen MS, Lundbye-Jensen J, Geertsen SS, Petersen TH, Paulson OB, Nielsen JB. Premotor cortex modulates somatosensory cortex during voluntary movements without proprioceptive feedback. Nat Neurosci. 2007;10:417-9.

Cramer SC, Lastra L, Lacourse MG, Cohen MJ. Brain motor system function after chronic, complete spinal cord injury. Brain. 2005;128:2941-50.

Crone NE, Miglioretti DL, Gordon B, Lesser RP. Functional mapping of human sensorimotor cortex with electrocorticographic spectral analysis. II. Event-related synchronization in the gamma band. Brain. 1998;121:2301-15.

Dale AM, Fischl B, Sereno MI. Cortical surface-based analysis. I. Segmentation and surface reconstruction. Neuroimage. 1999;9:179-94.

Duhamel JR, Goldberg ME, Fitzgibbon EJ, Sirigu A, Grafman J. Saccadic dysmetria in a patient with a right frontoparietal lesion. The importance of corollary discharge for accurate spatial behaviour. Brain. 1992;115 1387-402.

Farrer C, Franck N, Georgieff N, Frith CD, Decety J, Jeannerod M. Modulating the experience of agency: a positron emission tomography study. NeuroImage. 2003;18:324-33.

Felleman DJ, Van Essen DC. Distributed hierarchical processing in the primate cerebral cortex. Cereb Cortex. 1991;1:1-47.

Fetz EE, Finocchio DV, Baker MA, Soso MJ. Sensory and motor responses of precentral cortex cells during comparable passive and active joint movements. J Neurophysiol. 1980;43:1070-89.

Flanagan JR, Vetter P, Johansson RS, Wolpert DM. Prediction Precedes Control in Motor Learning. Curr Biol. 2003;13:146-50.

Ford JM, Mathalon DH, Heinks T, Kalba S, Faustman WO, Roth WT. Neurophysiological evidence of corollary discharge dysfunction in schizophrenia. The American journal of psychiatry. 2001;158:2069-71. Fried I, Mukamel R, Kreiman G. Internally generated preactivation of single neurons in human medial frontal cortex predicts volition. Neuron. 2011;69:548-62.

Gaymard B, Rivaud S, Amarenco P, Pierrot-Deseilligny C. Influence of visual information on cerebellar saccadic dysmetria. Ann Neurol. 1994;35:108-12.

Goupillaud P, Grossmann A, Morlet J. Cycle-octave and related transforms in seismic signal analysis. Geoexploration. 1984;23:85-102.

Gritsenko V, Krouchev NI, Kalaska JF. Afferent input, efference copy, signal noise, and biases in perception of joint angle during active versus passive elbow movements. J Neurophysiol. 2007;98:114054.

Helmholtz HV. Treatise On Physiological Optics. New York: Dover Publications, Inc.; 2005. 
Hermes D, Miller KJ, Noordmans HJ, Vansteensel MJ, Ramsey NF. Automated electrocorticographic electrode localization on individually rendered brain surfaces. J Neurosci Methods. 2010;185:293-8. Hotz-Boendermaker S, Funk M, Summers P, Brugger P, Hepp-Reymond M-C, Curt A, et al. Preservation of motor programs in paraplegics as demonstrated by attempted and imagined foot movements.

Neuroimage. 2008;39:383-94.

Hughes G, Waszak F. ERP correlates of action effect prediction and visual sensory attenuation in voluntary action. Neuroimage. 2011;56:1632-40.

Ikeda a, Shibasaki H, Luders H. Cortical mapping using evoked potentials and Bereitschafts potentials:

Textbook of Epilepsy Surgery. London: Informa Healthcare; 2008.

Johansson RS, Cole KJ. Sensory-motor coordination during grasping and manipulative actions. Curr Opin Neurobiol. 1992;2:815-23.

Johnson LA, Wander JD, Sarma D, Su DK, Fetz EE, Ojemann JG. Direct electrical stimulation of the somatosensory cortex in humans using electrocorticography electrodes: a qualitative and quantitative report. J Neural Eng. 2013;10:036021.

Johnson PB, Ferraina S, Bianchi L, Caminiti R. Cortical networks for visual reaching: physiological and anatomical organization of frontal and parietal lobe arm regions. Cereb Cortex. 1991;6:102-19.

Jordan MI, Rumelhart DE. Forward Models: Supervised Learning with a Distal Teacher. Cognitive Sci. 1992;16:307-54.

Kawato M, Kuroda T, Imamizu H, Nakano E, Miyauchi S, Yoshioka T. Internal forward models in the cerebellum: fMRI study on grip force and load force coupling. Prog Brain Res. 2003;142:171-88.

Kubánek J, Miller KJ, Ojemann JG, Wolpaw JR, Schalk G. Decoding flexion of individual fingers using electrocorticographic signals in humans. J Neural Eng. 2009;6:066001.

Legatt AD, Kader A. Topography of the initial cortical component of the median nerve somatosensory evoked potential. Relationship to central sulcus anatomy. J Clin Neurophysiol. 2000;17:321-5.

Leiguarda RC, Marsden CD. Limb apraxias: higher-order disorders of sensorimotor integration. Brain. 2000;123:860-79.

Mesgarani N, Chang EF. Selective cortical representation of attended speaker in multi-talker speech perception. Nature. 2012;485:233-6.

Miall RC, Wolpert DM. Forward Models for Physiological Motor Control. Neural Networks. 1996;9:1265-79.

Miller KJ, Leuthardt EC, Schalk G, Rao RPN, Anderson NR, Moran DW, et al. Spectral changes in cortical surface potentials during motor movement. J Neurosci. 2007;27:2424-32.

Moritz CT, Fetz EE. Volitional control of single cortical neurons in a brain-machine interface. J Neural Eng. 2011;8:025017.

Nelson RJ. Activity of monkey primary somatosensory cortical neurons changes prior to active movement. Brain Res. 1987;406:402-7.

Nichols TE. Multiple testing corrections, nonparametric methods, and random field theory. Neuroimage. 2012;62:811-5.

Nicolelis MaL, Lebedev Ma. Principles of neural ensemble physiology underlying the operation of brainmachine interfaces. Nat Rev Neurosci. 2009;10:530-40.

Nijboer F, Furdea A, Gunst I, Mellinger J, McFarland DJ, Birbaumer N, et al. An auditory braincomputer interface (BCI). J Neurosci Methods. 2008;167:43-50.

Nishitani N, Hari R. Temporal dynamics of cortical representation for action. Proc Natl Acad Sci U S A. 2000;97:913-8.

Nishitani N, Hari R. Viewing lip forms: cortical dynamics. Neuron. 2002;36:1211-20.

Nur D, Wol RC. Phase randomisation : numerical study of higher cumulants behaviour. Comput Stat Data An. 2001;37:487-513.

Pei X, Leuthardt EC, Gaona CM, Brunner P, Wolpaw JR, Schalk G. Spatiotemporal dynamics of electrocorticographic high gamma activity during overt and covert word repetition. Neuroimage. 2011;54:2960-72. 
Penny W, Friston K, Ashburner J, Kiebel S, Nichols T. Statistical Parametric Mapping: The Analysis of Functional Brain Images 1st ed: Academic Press; 2006.

Poulet JF, Hedwig B. A corollary discharge maintains auditory sensitivity during sound production. Nature. 2002;418:872-6.

Ramos-Murguialday A, Schürholz M, Caggiano V, Wildgruber M, Caria A, Hammer EM, et al. Proprioceptive feedback and brain computer interface (BCI) based neuroprostheses. PLoS One. 2012;7:e47048.

Ray S, Crone NE, Niebur E, Franaszczuk PJ, Hsiao SS. Neural correlates of high-gamma oscillations (60$200 \mathrm{~Hz}$ ) in macaque local field potentials and their potential implications in electrocorticography. J Neurosci. 2008;28:11526-36.

Roy JE, Cullen KE. Selective processing of vestibular reafference during self-generated head motion. J Neurosci. 2001;21:2131-42.

Sato Y, Fukuda M, Oishi M, Fujii Y. Movement-related cortical activation with voluntary pinch task: simultaneous monitoring of near-infrared spectroscopy signals and movement-related cortical potentials. J Biomed Opt. 2012;17:076011.

Schalk G, McFarland DJ, Hinterberger T, Birbaumer N, Wolpaw JR. BCI2000: a general-purpose braincomputer interface (BCI) system. IEEE transactions on bio-medical engineering. 2004;51:1034-43.

Schwartz AB, Cui XT, Weber DJ, Moran DW. Brain-controlled interfaces: movement restoration with neural prosthetics. Neuron. 2006;52:205-20.

Seki K, Fetz EE. Gating of sensory input at spinal and cortical levels during preparation and execution of voluntary movement. J Neurosci. 2012;32:890-902.

Shoham S, Halgren E, Maynard EM, Normann RA. Motor-cortical activity in tetraplegics. Nature. 2001;413:793.

Sommer Ma, Wurtz RH. A pathway in primate brain for internal monitoring of movements. Science. 2002a;296:1480-2.

Sommer Ma, Wurtz RH. A pathway in primate brain for internal monitoring of movements. Science (New York, NY). 2002b;296:1480-2.

Soso MJ, Fetz EE. Responses of identified cells in postcentral cortex of awake monkeys during comparable active and passive joint movements. J Neurophysiol. 1980;43:1090-110.

Swann NC, Tandon N, Pieters TA, Aron AR. Intracranial electroencephalography reveals different temporal profiles for dorsal- and ventro-lateral prefrontal cortex in preparing to stop action. Cereb Cortex. 2013;23:2479-88.

Talairach J, Tournoux P. Co-planar stereotaxic atlas of the human brain: 3-dimensional proportional system: an approach to cerebral imaging. Neuropsychologia. 1988;39:145.

Tsakiris M, Prabhu G, Haggard P. Having a body versus moving your body: How agency structures bodyownership. Consciousness and cognition. 2006;15:423-32.

Tsubokawa T, Katayama Y, Yamamoto T, Hirayama T, Koyama S. Chronic motor cortex stimulation for the treatment of central pain. Acta Neurochir Suppl (Wien). 1991;52:137-9.

van Albada SJ, Robinson Pa. Transformation of arbitrary distributions to the normal distribution with application to EEG test-retest reliability. J Neurosci Methods. 2007;161:205-11.

von Holst E. Relations between the central Nervous System and the peripheral organs. Anim Behav. 1954;2:89-94.

Weinrich M, Wise SP, Mauritz KH. A neurophysiological study of the premotor cortex in the rhesus monkey. Brain : a journal of neurology. 1984;107 ( Pt 2):385-414.

Wise SP, Boussaoud D, Johnson PB, Caminiti R. Premotor and parietal cortex: corticocortical connectivity and combinatorial computations. Annu Rev Neurosci. 1997;20:25-42.

Wolpert DM, Ghahramani Z, Jordan MI. An internal model for sensorimotor integration. Science. 1995;269:1880-2.

Yoshor D, Ghose GM, Bosking WH, Sun P, Maunsell JHR. Spatial attention does not strongly modulate neuronal responses in early human visual cortex. J Neurosci. 2007;27:13205-9. 


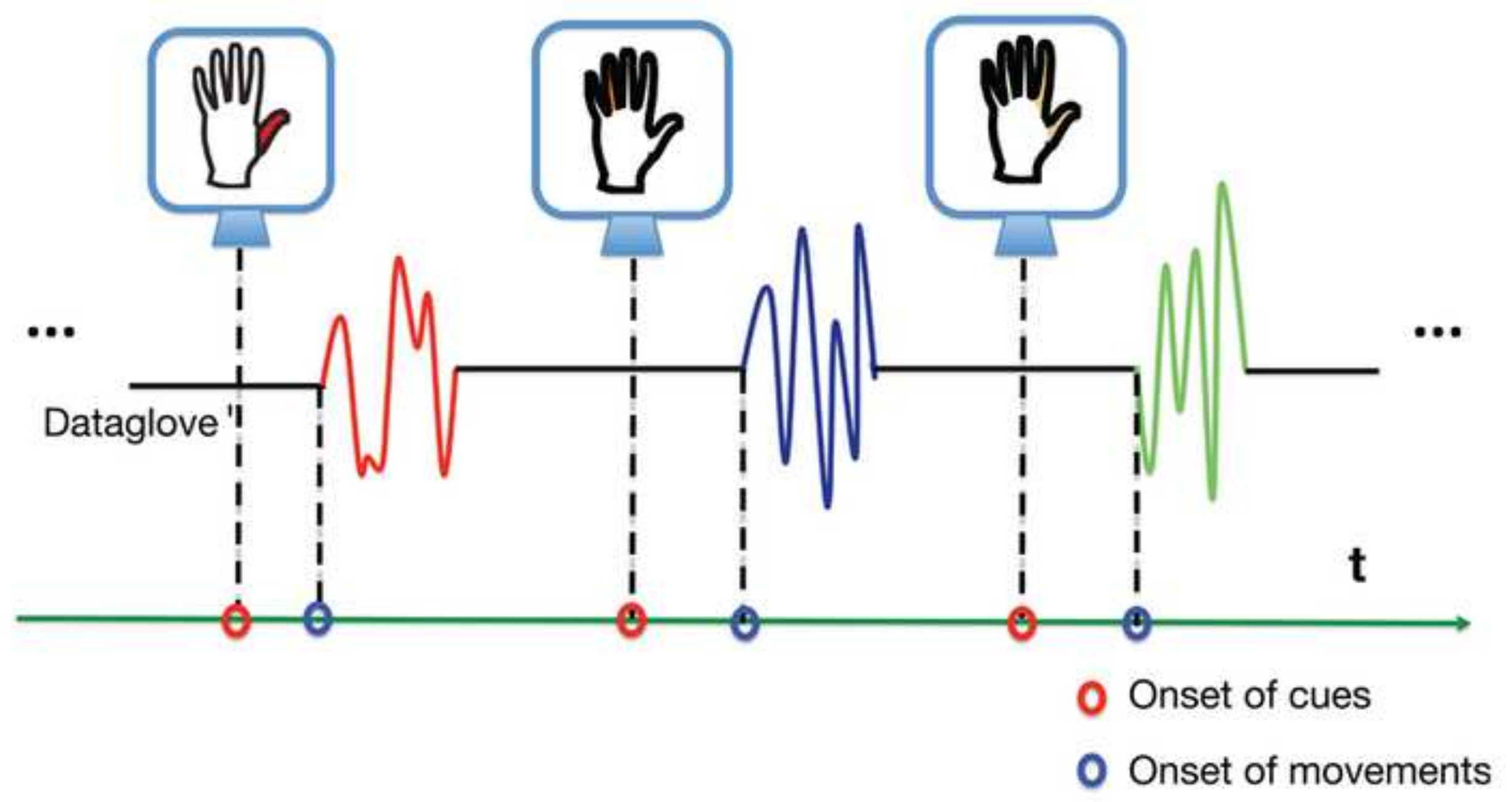


Subjects:

10

20

3 口

4 이

5 ㅁ

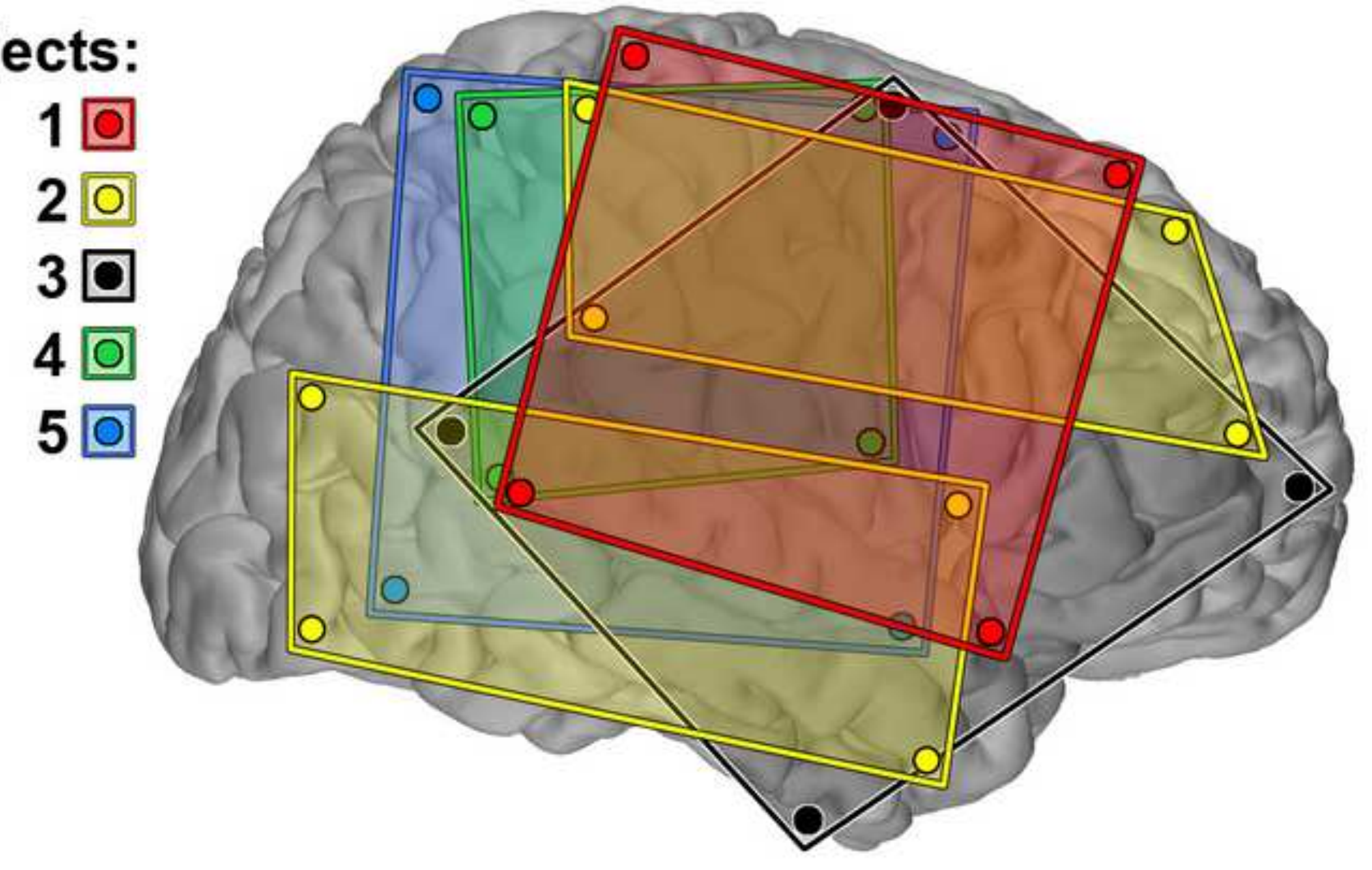



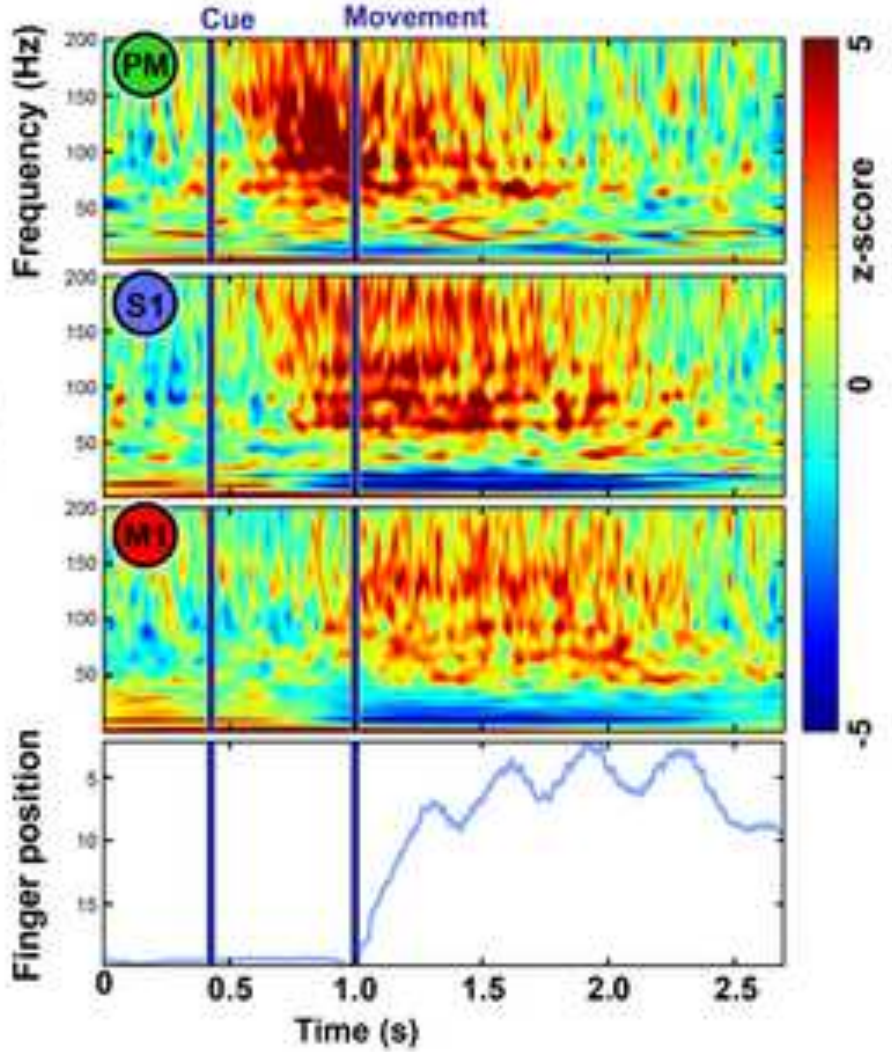

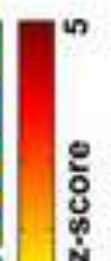

.
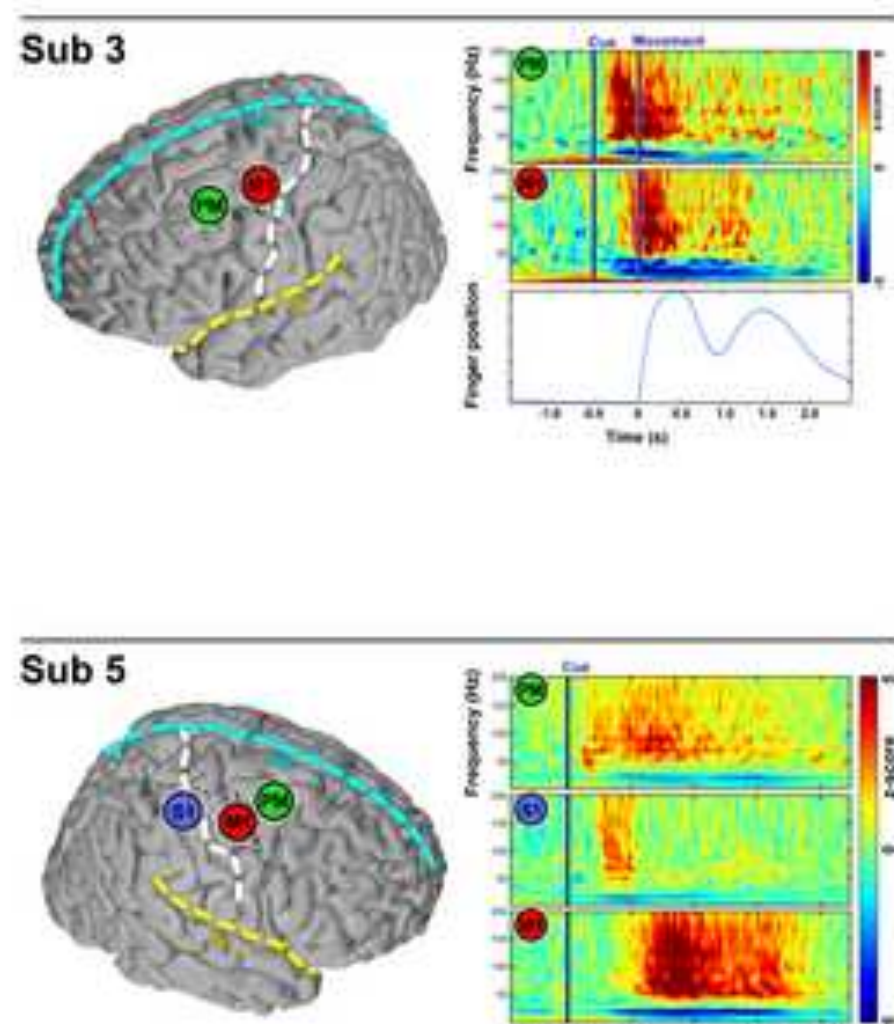

\section{Figure 3}

Sub 2

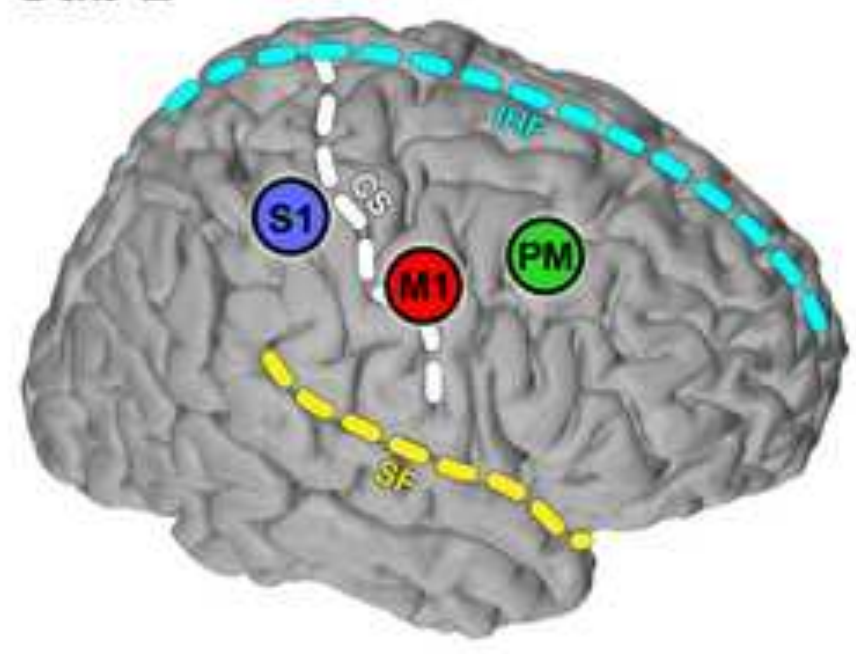

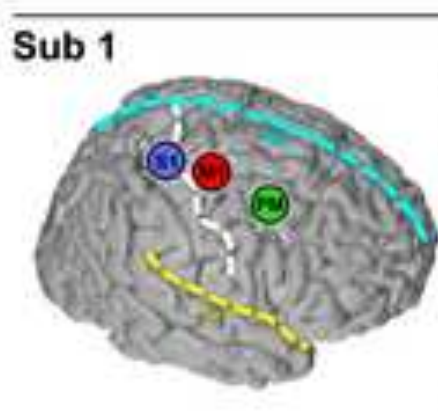

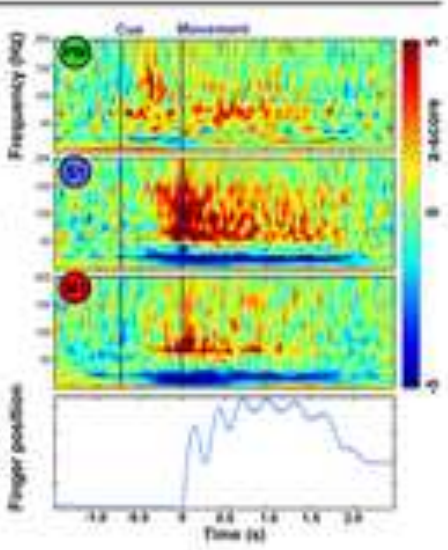

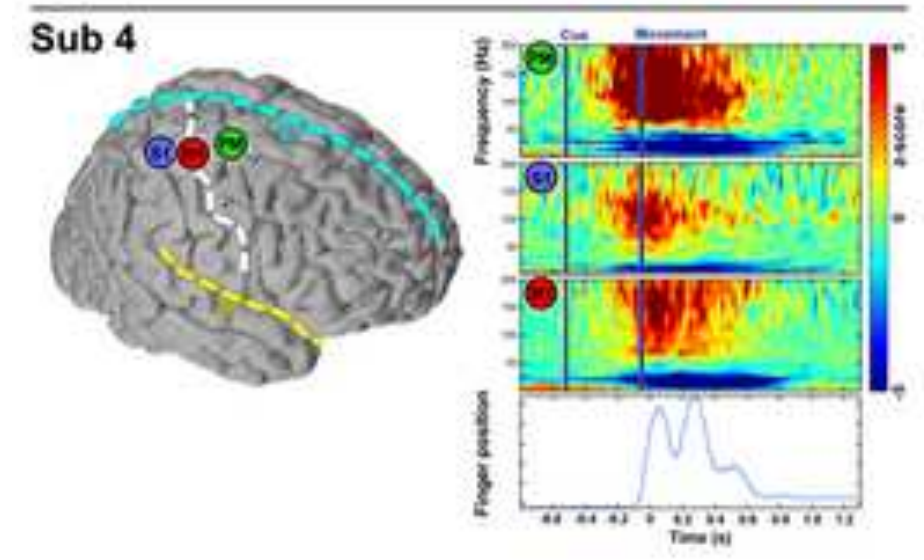

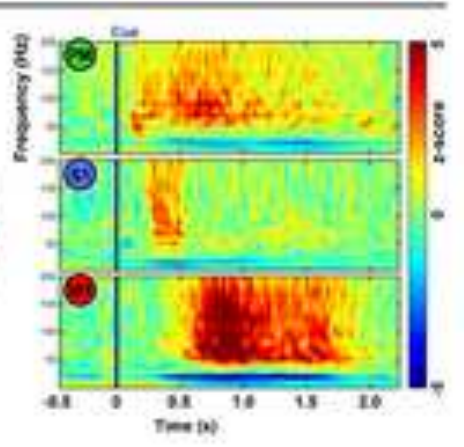


Figure 4
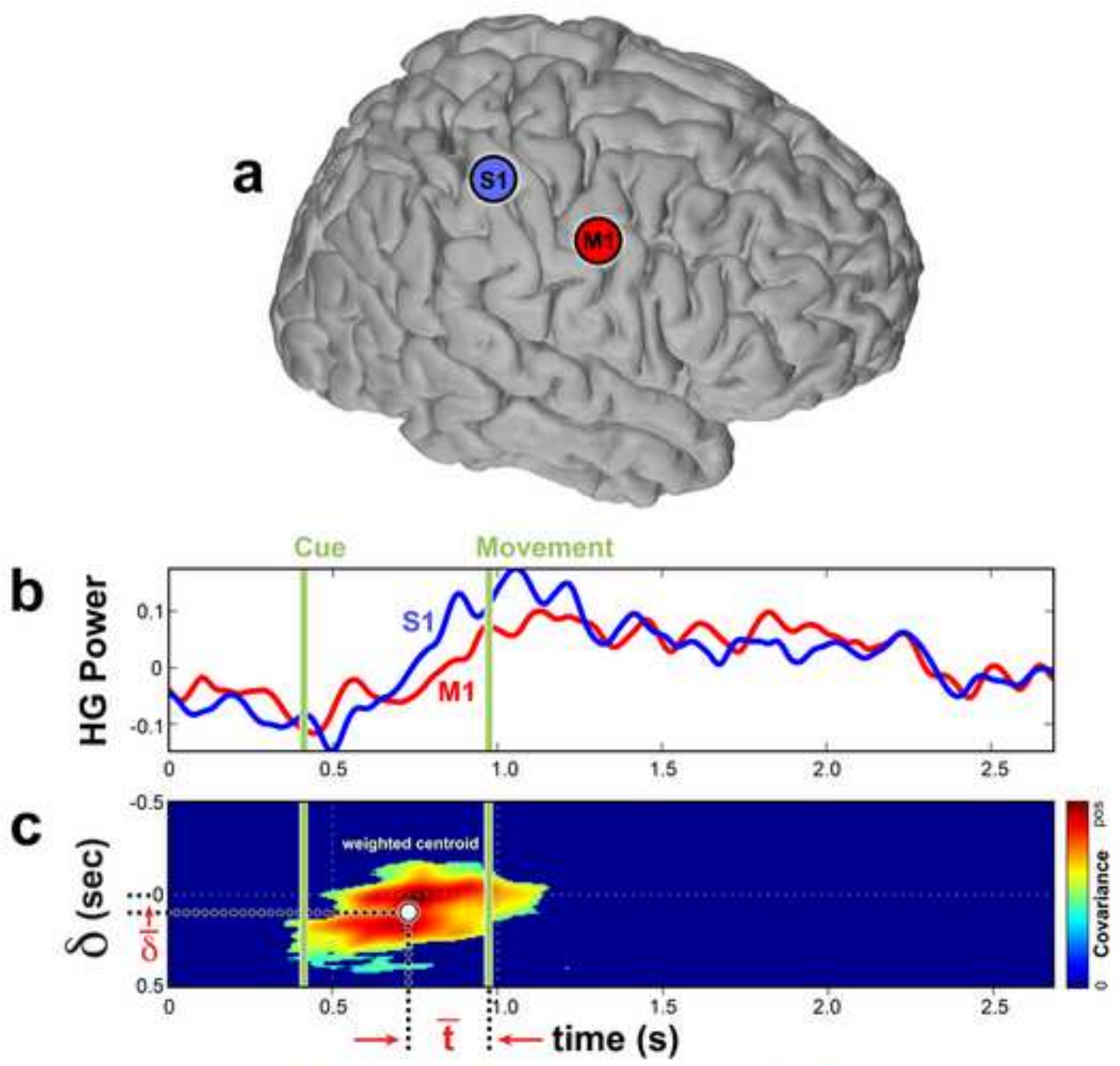

d

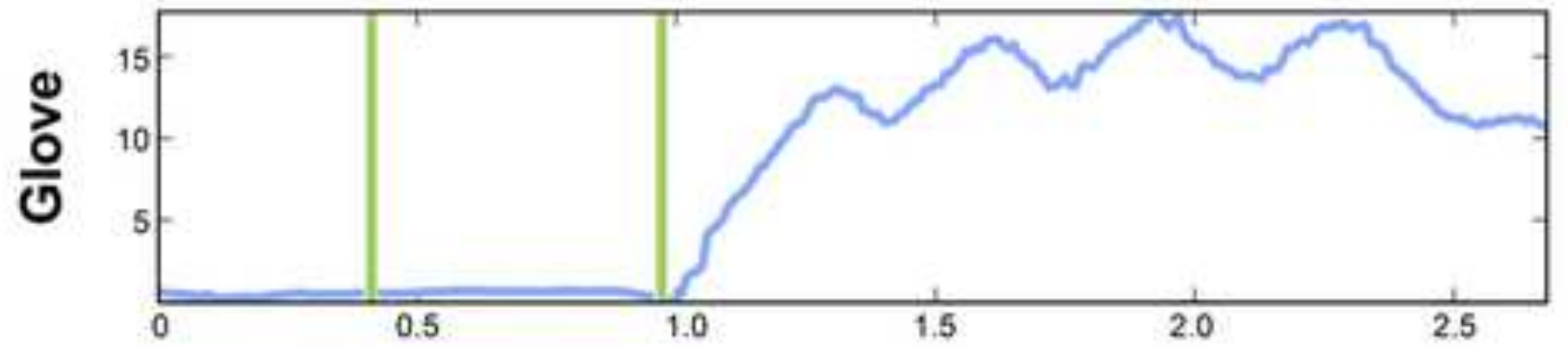


Sub 2

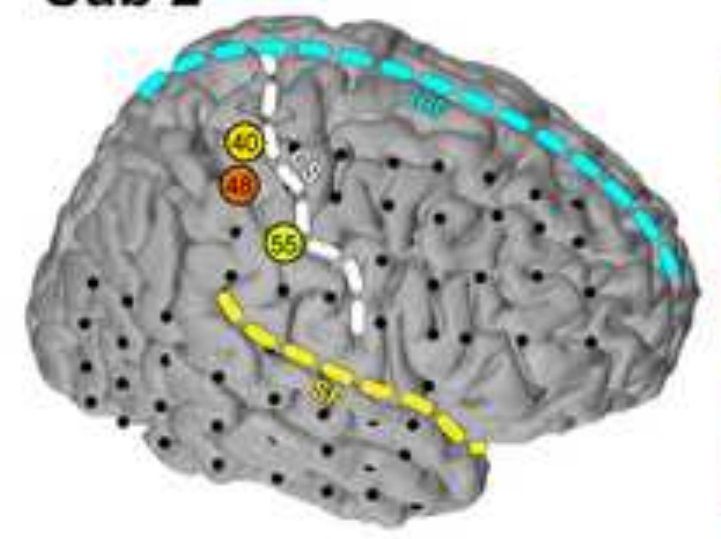

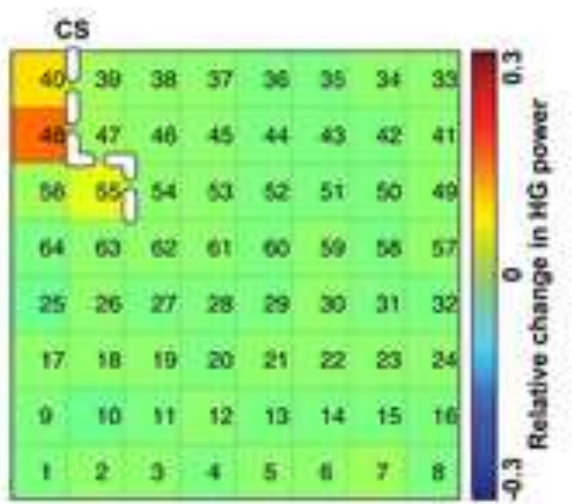

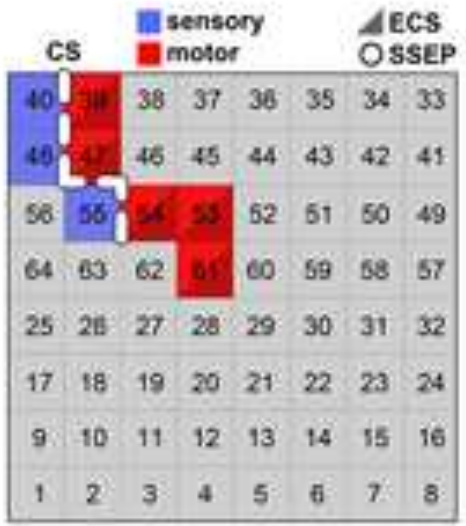

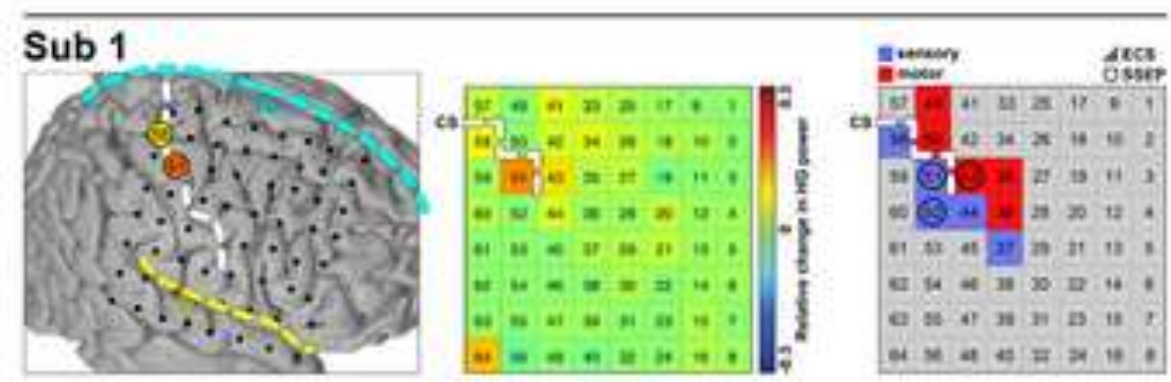

\section{Sub 3}
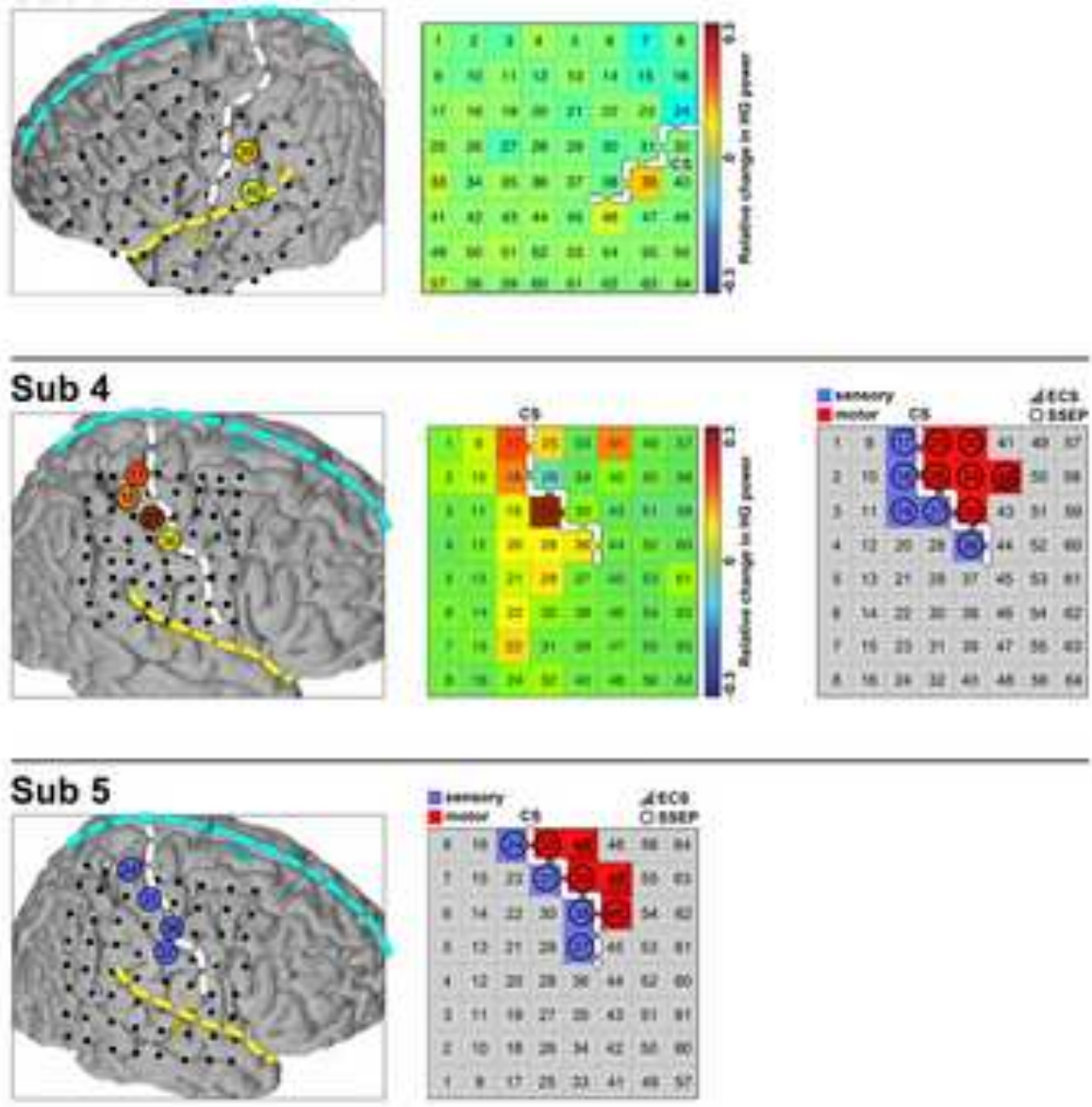
Figure 6

a

\begin{tabular}{|c|c|c|c|}
\hline & \multicolumn{3}{|c|}{ Electrodes (n) } \\
\hline Subject & PM • & S1 & M1 A \\
\hline $\mathbf{1}$ & 3 & 6 & 6 \\
\hline $\mathbf{2}$ & 3 & 5 & 5 \\
\hline 3 & 4 & 0 & 1 \\
\hline $\mathbf{4}$ & 3 & 4 & 7 \\
\hline $\mathbf{5}$ & 1 & 4 & 5 \\
\hline
\end{tabular}
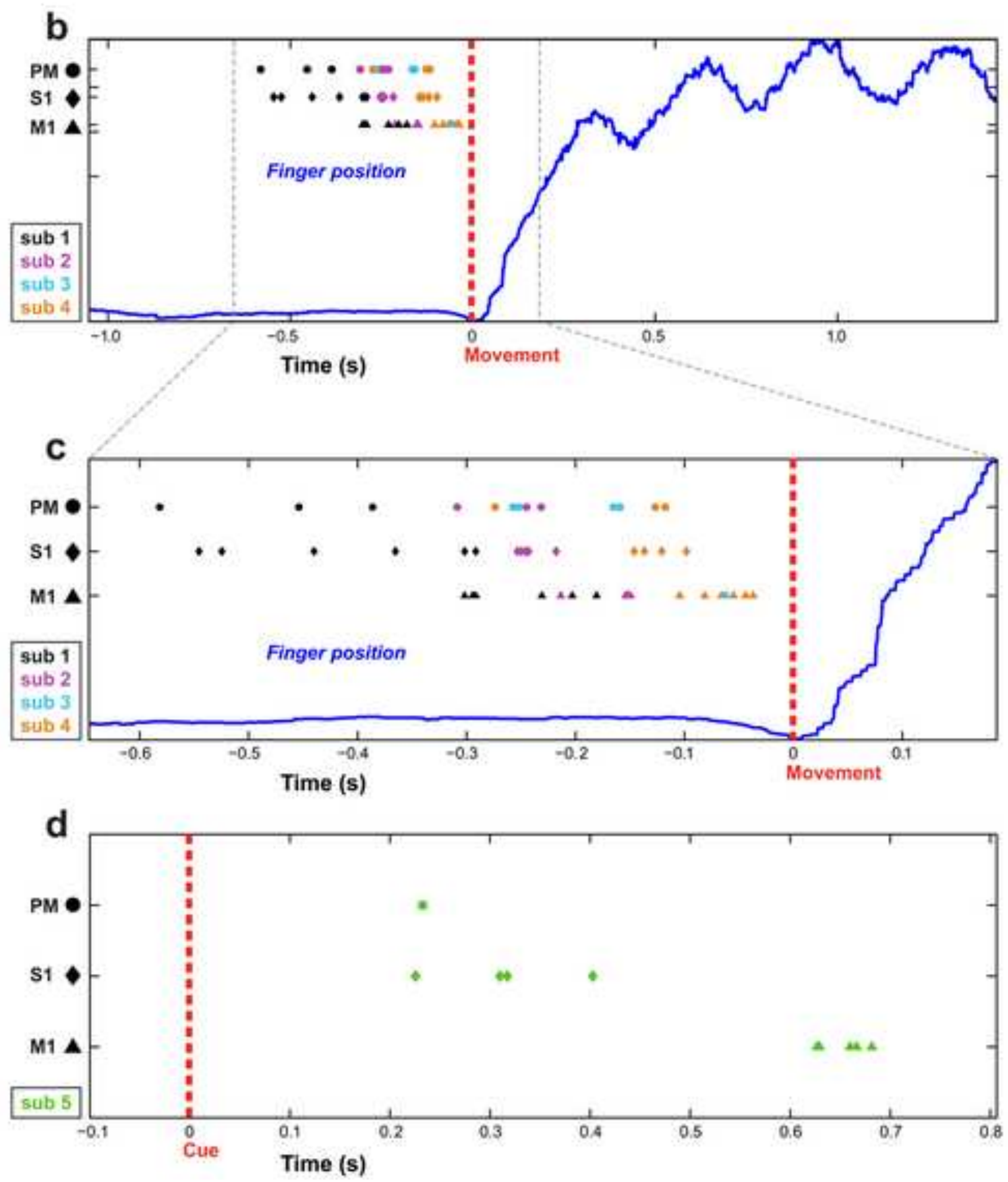


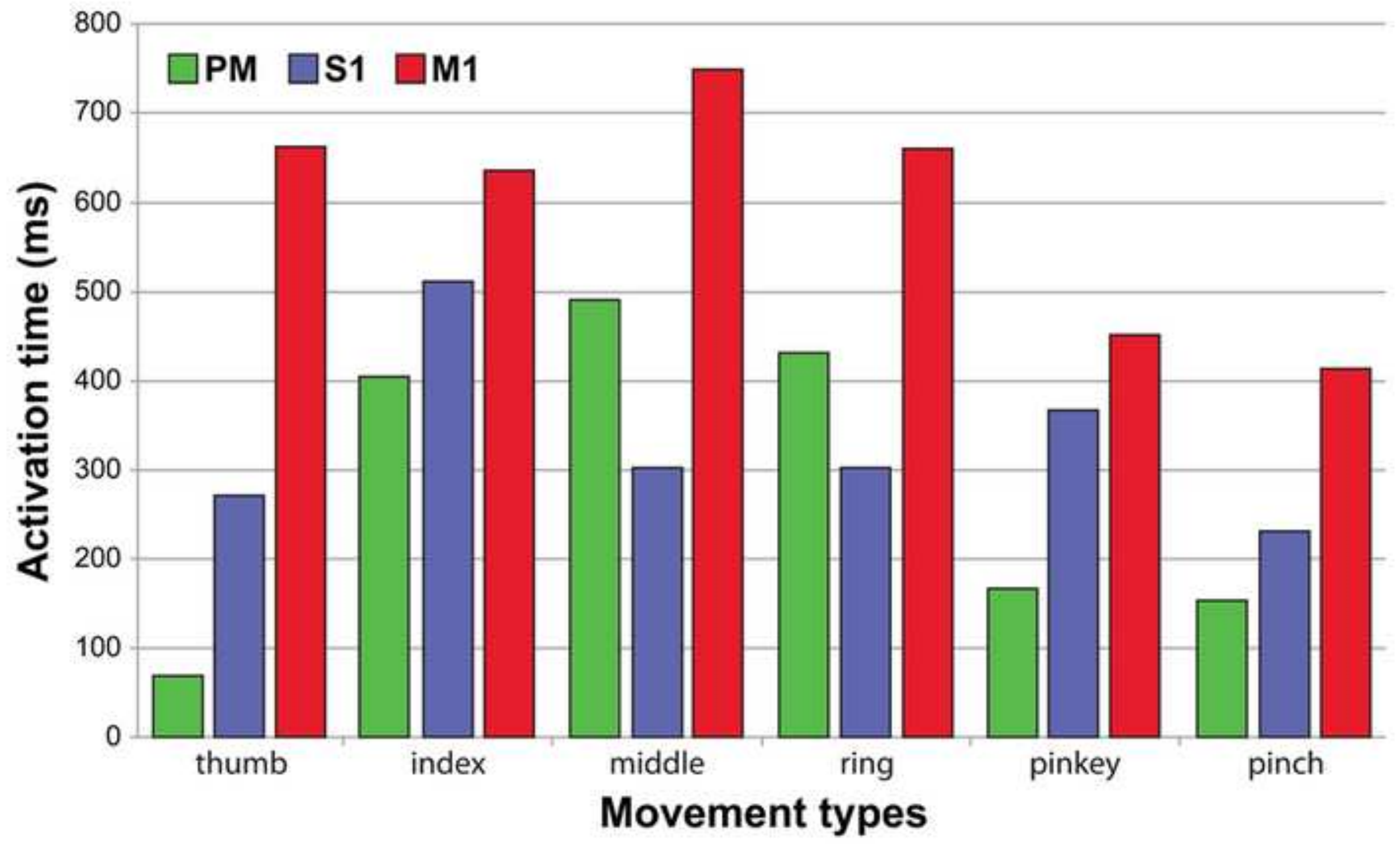



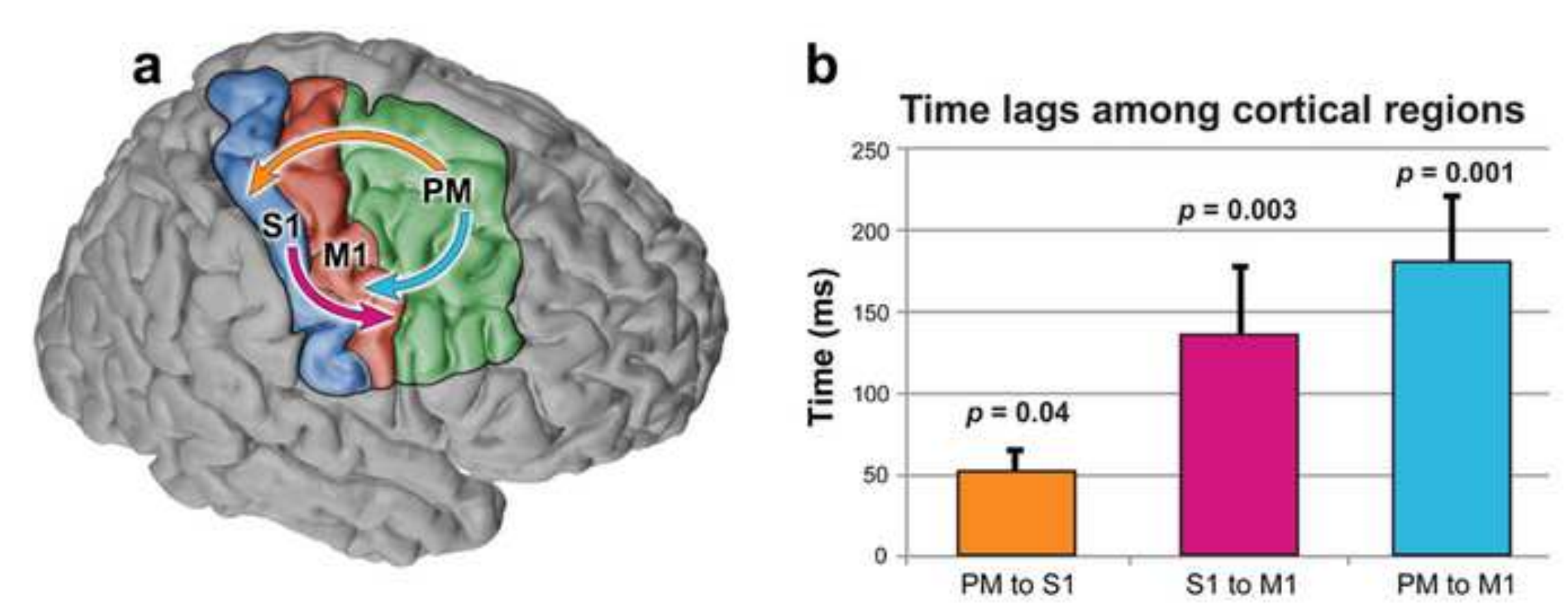
Table 1. Study subject demographics.

\begin{tabular}{|c|c|c|c|}
\hline & \multicolumn{3}{|c|}{ Subject demographics } \\
\hline Subject & Gender & Age (years) & Seizure etiology \\
\hline 1 & male & 20 & Post-trauma \\
\hline 2 & male & 54 & Post-trauma \\
\hline 3 & female & 45 & Focal cortical dysplasia \\
\hline 4 & male & 18 & Vascular malformation \\
\hline 5 & male & 20 & Focal cortical dysplasia \\
\hline
\end{tabular}

Table 2. Means and standard errors of the means (SE) of the $\mathrm{HG}$ activation time ( $\mathrm{ms} \pm \mathrm{SE}$ ) of the electrodes localized to each cortical region.

\begin{tabular}{|c|c|c|c|}
\hline & \multicolumn{3}{|c|}{ region HG activation time (ms \pm SE) } \\
\hline Subject & PM & S1 & M1 \\
\hline 1 & $-474 \pm 57$ & $-411 \pm 45$ & $-250 \pm 21$ \\
\hline 2 & $-261 \pm 24$ & $-241 \pm 6$ & $-163 \pm 13$ \\
\hline 3 & $-209 \pm 26$ & & -62 \\
\hline 4 & $-172 \pm 50$ & $-126 \pm 11$ & $-59 \pm 12$ \\
\hline 5 & 233 & $314 \pm 36$ & $653 \pm 11$ \\
\hline
\end{tabular}

Subject 3 had no S1 electrodes. SE was not computed for the results obtained with only one electrode. 